\title{
Entre redes, patrimonio y turismo: mutaciones de la pesca artesanal en la costa norte de Barcelona (Cataluña)
}

\section{Between nets, heritage and tourism: mutations in artisanal fishing at the northern coast of Barcelona (Catalonia)}

Eliseu Carbonell $^{1}$ http://orcid.org/0000-0003-0335-4421

${ }^{1}$ Universitat de Girona, Departamento de Pedagogía, Girona, ESPAÑA. Email: eliseu. carbonell@udg.edu

\begin{abstract}
Resumen
En lo que llevamos de siglo XXI en Cataluña hemos visto reducirse a la mitad la flota pesquera artesanal. En el mismo período Cataluńa se ha consolidado como la segunda región europea en importancia turística por detrás de la Región Parisina. Al mismo tiempo, otro fenómeno cultural ha irrumpido en las costas catalanas estos últimos ańos: la revitalización de las tradiciones marítimo-pesqueras a través del patrimonio cultural. Observamos cómo a lo largo de la costa se recuperan embarcaciones tradicionales, se realizan demostraciones de sistemas de pesca caídos en desuso, se revaloriza la gastronomía tradicional marinera, etcétera. Todo ello abre un campo de oportunidades para aquellos pescadores que se ven obligados a abandonar su actividad. Observando la pesca en Cataluña en una dimensión histórica, nos damos cuenta de que a lo largo del tiempo el número de pescadores oscilaba en función del contexto social y económico. Podemos preguntarnos si el momento actual de crisis, con la desaparición de un tercio de los puestos de trabajo en la pesca en lo que llevamos de siglo, no será más que un bache temporal favorecido por el apogeo turístico, o si por el contrario el patrimonio marítimo renovará el crecimiento turístico hasta la extinción de la pesca artesanal.
\end{abstract}

Palabras claves: pesca artesanal, patrimonio marítimo, turismo, Cataluña.

\begin{abstract}
In the first decades of the $21^{\text {st }}$ century, the artisanal fishing fleet in Catalonia reduced by half. In the same period Catalonia consolidated as the second European region in tourism importance behind the Parisian Region. Meanwhile, another cultural phenomenon appeared on the Catalan coasts, the revitalization of maritime-fishing traditions through cultural heritage. One can see how along the coast traditional boats are recovered, demonstrations of abandoned fishing systems are performed, traditional fishing gastronomy is revalorized, etc. This phenomenon brings a field of opportunities for those fishermen who are forced to abandon their jobs. By looking at fishing in Catalonia in historical perspective, one realizes that over time the number of fishermen oscillated according to the social and economic context. One can therefore ask whether the present moment of crisis, with the disappearance of a third of the jobs in fishing, will be nothing more than a passenger pothole favoured by the tourist heyday, or if contrarily the maritime heritage will renew the tourism growth until the disappearance of artisanal fishing.
\end{abstract}

Keywords: artisanal fishing, maritime heritage, tourism, Catalonia.

Recibido: 21 julio 2017. Aceptado: 18 abril 2018 


\section{Introducción}

En el año 2004 se celebró en Barcelona el "Fórum Universal de las Culturas", que pretendía ser un evento que emulara los Juegos Olímpicos de Barcelona 92 en el sentido de atraer la atención mundial sobre una ciudad que había apostado decididamente por una economía basada en el turismo y los servicios. Esta política planificada desde el Ayuntamiento y la Cámara de Comercio de Barcelona situaría a Cataluña como una de las principales regiones turísticas del Mediterráneo, Europa y el mundo; la segunda de Europa por detrás de la región francesa de Île-de-France (Palou, 2012, p. 424). La irrupción de nuevos medios de transporte como los vuelos "low-cost" y el auge de los cruceros en el puerto de Barcelona ayudarán a este impulso del turismo en Cataluña a inicios del siglo XXI (Garay y Cànoves, 2010, p. 54). Pero este modelo de desarrollo que se llegó a llamar "Modelo Barcelona" sería objeto de fuerte crítica por parte de muchos intelectuales catalanes (ver particularmente Delgado, 2005). Curiosamente, aquel mismo año 2004 el Ayuntamiento de Barcelona encargaría a Perejaume (artista plástico, ensayista y poeta nacido en Sant Pol de Mar en 1957) la elaboración del cartel anunciante de las "Festes de la Mercè" (Fiesta Mayor de Barcelona). El artista, cuya obra contiene en buena medida una reflexión crítica sobre la pérdida de la cultura agraria del país, realizó un provocador cartel con tres preguntas inscritas sobre un pedestal, la última de las cuales decía: "Pot tornar a pagès aquell que ha estat turista?". ${ }^{1}$ No hace falta decir que el cartel de las fiestas levantó una gran polvareda en los medios de comunicación. Perejaume vive en Sant Pol de Mar, un pequeño pueblo de la costa norte de Barcelona que aparecerá más adelante en este artículo, cuya economía tradicional hasta la llegada del turismo se repartía entre la agricultura y la pesca. De hecho, según Alegret y Nadal (1987), Sant Pol de Mar fue el pueblo de mayor importancia pesquera de la comarca en la primera mitad del siglo XX. De manera

1 Traducción: "¿Puede volver al campesinado quien ha sido turista?". "Pagès", en castellano "payés", es según la RAE el nombre que recibe el campesino en Cataluńa y Baleares. Pero no solo designa al campesino sino también su lugar y modo de vida, de tal manera que en esta frase "tornar a pagès" significa literalmente volver al campo o volver al modo de vida campesino. que Perejaume podría haber intercambiado perfectamente "pagès" por "pescador".

El propósito de este artículo es exponer la situación de la pesca artesanal en Cataluña y concretamente en la costa norte de Barcelona, la comarca de El Maresme. Para hacerlo, voy en primer lugar a ofrecer una breve mirada sobre el contexto histórico y actual en el que se desarrolla la pesca en Cataluña. Si históricamente el pescador tenía que combinar este oficio con el de payés, en la actualidad el pescador catalán se ha tenido que ir acostumbrando a convivir con los turistas hasta el punto, en algunos casos, de entrar de lleno como actor en la esfera del turismo. La sobrepoblación de las costas provocada por el turismo es una de las causas de la degradación de los ecosistemas marinos. Ello, junto con otras causas endógenas a la pesca en las últimas décadas, como el desarrollo tecnológico y otros elementos que veremos más adelante, llevará a una situación de crisis generalizada del sector. Intuimos pues aquí un peligroso círculo.

Esto coincide en el tiempo con otro fenómeno importante que surge en las costas catalanas a principios del siglo XXI y es una especie de despertar patrimonial o, como diría Jeremy Boissevain (1992), una especie de revitalización de tradiciones que durante algunas décadas se habían mantenido durmientes y que repentinamente son restauradas o resucitadas. Ello tiene que ver también con el impulso que recibe el patrimonio de los oficios tradicionales tras la Convención de la UNESCO de 2003 para la Salvaguarda del Patrimonio Inmaterial que da combustible a muchos museos y otros programadores culturales de las administraciones locales. Repasaremos aquí los principales resultados de una investigación sobre los procesos de patrimonialización del ámbito marítimo-pesquero llevada a cabo por un equipo de investigadores que tuve el privilegio de coordinar como IP (Proyecto PESCUM) entre 2011 y 2013, para darnos cuenta del peso del patrimonio marítimo, sobre todo el patrimonio inmaterial, y el panorama que a partir de esta realidad se abre para la pesca artesanal.

Como corolario expondré la situación personal de dos pescadores del puerto de Arenys de Mar (Barcelona) con los que llevo ańos conversando de manera formal e informal sobre la pesca, en el marco de mi 
trabajo de campo etnográfico sobre la patrimonialización del ámbito marítimo-pesquero. Aunque generalizar siempre es arriesgado, su testimonio me parece una buena ejemplificación de las transformaciones que están aconteciendo actualmente en la pesca artesanal en la costa norte de Barcelona y, por extensión, en Cataluña y probablemente otras regiones de la orilla norte del Mediterráneo.

Antes de dar paso a la primera sección del artículo, a continuación situaré brevemente el marco teórico en el que se desarrolla este trabajo. A pesar de que existen algunos precedentes de estudios etnológicos, como los trabajos de Roig (1927) y Amades (1936), la antropología de la pesca no empieza a desarrollarse en Cataluña sino hasta los años ochenta con trabajos enmarcados en los estudios de comunidad característicos de la antropología ibérica de aquel momento (Prat, Martínez, Contreras, 1991, p. 118). En este sentido cabe mencionar como primera etnografía completa centrada en la pesca artesanal en Cataluña el trabajo de Joan Lluís Alegret (1986) sobre los "armelladers" de Palamós. Como exponen Pascual y Alegret (2004), es a partir de los años ochenta cuando se desarrollará en el Estado español una antropología de la pesca interesada básicamente en tres temáticas: interacción entre turismo y pesca, modelos de gestión, y perspectiva de género. Más recientemente habrá que añadir una cuarta temática, los procesos de patrimonialización marítimopesqueros que toman importancia, como veremos, con la entrada al siglo actual.

En el ámbito geográfico que nos ocupa, la costa catalana, la interacción entre pesca y turismo fue ya tratada por Pi i Sunyer (1977a) en capítulo dentro de un volumen sobre antropología del turismo, pero hasta el día de hoy ha sido poco trabajado por la antropología en Cataluña salvo algunas excepciones (De la Cruz, 2012; Palou, 2017). El segundo gran tema que ha ocupado a la antropología de la pesca en Cataluña han sido los modos de apropiación y gestión de las áreas de pesca. Los primeros trabajos analizaron zonas de gestión consuetudinaria como la pesca en las zonas lacustres del delta del Ebro (Andreu, 1981, 1985). También aparecen trabajos que se interesan por los conflictos en la gestión de la pesca entre distintas modalidades de pesca (Pi i Sunyer, 1977b; Breton, 1987), así como particularmente los estudios de
Alegret sobre los modelos de cogestión en Cataluña (Alegret, 1998, 1999a, 1999b). En tercer lugar, la perspectiva de género ha estado también presente en los estudios de antropología de la pesca en Cataluña, centrados en aspectos concretos del proceso de la pesca realizados tradicionalmente por mujeres como la reparación de redes (Alberich, 2001; Pujol y Carbonell, 2009), o bien trabajos que ponen de manifiesto la invisibilidad de las mujeres en el ámbito pesquero (Breton, Lladó y Badia, 1992; Torra y Carbonell, 2015). Finalmente, en los últimos años se ha abierto un campo de estudio que está generando nuevas investigaciones y publicaciones que tratan sobre la patrimonialización del ámbito marítimopesquero que representa a la vez una oportunidad y un desafío para los pescadores. Una muestra de ello son los trabajos de Carbonell $(2013,2014)$ y Alegret (2016a) entre otros. El presente artículo se sitúa en este marco teórico de la patrimonialización, a pesar de entroncar con otro de los temas que más han preocupado en las últimas décadas a la antropología de la pesca en España, como son las relaciones complejas y ambivalentes entre la pesca y el turismo.

\section{Contexto de la pesca en Cataluńa}

En este apartado se ofrece una breve descripción del contexto histórico, así como algunos datos fundamentales sobre la situación actual de la pesca en Cataluña para poder entender el marco actual en el que se desarrolla esta actividad.

\section{Contexto histórico: pescadores y payeses}

Fernand Braudel ya había puesto de manifiesto que la escasez de recursos pesqueros del Mediterráneo, comparado con la producción de los océanos, obligó históricamente a sus habitantes a tener que asociar las economías marítimas con las terrestres para poder subsistir. La exigüidad de los alimentos de tierra empujó a los catalanes al mar, escribía Braudel: "Sabia fórmula de vida, residuo de viejas tradiciones marítimas del Mediterráneo, que combinan los escasos recursos de la tierra con los escasos recursos del mar" (Braudel, 1949, p. 111). La geógrafa Yvette Barbaza a su vez, enlazando con Braudel, escribirá sobre el paisaje humano de la Costa Brava anterior a la llegada del turismo masivo: 
"La fórmula del pescador-payés está perfectamente justificada a escala individual e incluso de grupo, ya que cada pescador joven tiende a convertirse en pescador-payés. La segunda actividad es escogida libremente, aunque impuesta por la necesidad. Pero es gratamente practicada y con placer. El pescador se siente ligado a su viñedo, a su huerto" (Barbaza, 1988, p. 286).

La modernización en la pesca en Cataluña se produce en la segunda mitad del siglo XVIII. Ello se explica por dos razones. En primer lugar a la intervención por primera vez del Estado en la gestión de la pesca a través de las Ordenanzas de Matrícula por el rey Carlos III en 1751, que son una copia de las promulgadas en Francia en 1668 (Llovet, 1984). Pero también, a la gran difusión que en Cataluña tendrá la introducción de nuevos sistemas de pesca intensiva como el palangre y el "art del bou" o arte de arrastre mediante una pareja de embarcaciones. De acuerdo con Alegret y Martí (2003, pp. 27-28), ya en aquel momento las Cofradías reaccionaron oponiéndose a la intervención del Estado y a la innovación tecnológica que suponía el "bou" con argumentos que hoy calificaríamos de ambientalistas, como por ejemplo que las nuevas artes de pesca "mataban a las crías sin dejarlas crecer".

Esta tensión la vamos reencontrando a lo largo de la historia moderna y contemporánea y hasta la actualidad. Los primeros trabajos antropológicos realizados en Cataluña en el ámbito de la pesca recogen testimonios frecuentes del enfrentamiento entre lo que, sobre todo a partir de los años ochenta, vendrá a llamarse los "artesanales" -pequeñas embarcaciones que forman la flota dedicada a las artes menores, como redes, palangres y nasas- versus los "industriales" -embarcaciones dedicadas al arrastre, al cerco y al palangre de fondo- (Pi i Sunyer, 1977b; Andreu, 1985; Alegret, 1986; Breton, 1987). Más adelante, cuando aportemos testimonios etnográficos, volveremos sobre esta cuestión.

De todos modos, los sistemas de pesca, después de las novedades introducidas en el siglo XVIII se mantuvieron con pocas alteraciones hasta el siglo $\mathrm{XX}$, cuando se producen una serie de innovaciones tecnológicas importantes. En primer lugar, la introducción del motor en las embarcaciones de pesca, que substituye la propulsión a vela latina en los años veinte. En segundo lugar, el desarrollo del transporte terrestre y la fabricación industrial de hielo en los años treinta. En tercer lugar, la aparición de nuevos materiales como el nilón para la fabricación de redes en los sesenta. Y finalmente la introducción de las nuevas tecnologías de navegación, comunicación y detección de bancos de peces en la década del setenta (Bas y Camprubí, 1980; Mas, 1994; Alegret y Martí, 2003; Alegret y Garrido, 2008; Garrido, 2012).

En resumen, como demostró Alfons Garrigo (2012) en su tesis doctoral, la historia de la pesca en Cataluña se ha caracterizado, al menos desde el siglo $\mathrm{XVI}$, por ser una actividad de refugio que se combina con otros oficios o incluso se tiende a abandonar si surgen alternativas en tierra; o bien, al contrario, aparece como alternativa en episodios de crisis. Así, el número de pescadores en la costa catalana oscila a lo largo de la historia, aumentado en épocas de crisis, como guerras o plagas agrícolas y disminuyendo cuando la situación se normaliza.

\section{Contexto actual: pescadores y turistas}

Como recuerda Joandomènec Ros (2016, p. 11), el eminente ecólogo catalán Ramon Margalef solía decir que el Mediterráneo "era un mar para turistas, no para pescadores", ya que sus aguas azules y transparentes son la constatación de su relativa pobreza en organismos del bacterioplancton y el fitoplancton. En Cataluña además, como explica Alegret (2002), debido a su alta salinidad, la poca intensidad de las corrientes marinas y la estrechez de la plataforma continental, el mar es relativamente pobre en recursos pesqueros, cosa que no ha permitido el desarrollo de una industria pesquera comparable a la de otras regiones del mundo. La flota pesquera catalana en 2010, según un estudio de Greenpeace (Varela y Ojeda, 2010, p. 8), con 1.054 barcos, representaba un $1,23 \%$ de la flota dentro de la UE y aproximadamente un $10 \%$ de la flota pesquera dentro del Estado español, a pesar de ser el 16\% de la población del conjunto de España ${ }^{2}$ y de que la economía catalana representa el 20\% del Producto Interior Bruto (PIB)

2 Instituto de Estadística de Cataluña. https://www. idescat.cat/pub/?id=aec\&n=245\&lang=es Consultado 3.01.2017. 
Tabla 1. Censo de la flota catalana por modalidad de pesca.

\begin{tabular}{|c|c|c|c|}
\hline \multicolumn{4}{|c|}{$\begin{array}{l}\text { Flota pesquera y capturas. 2015. Embarcaciones por modalidad. } \\
\text { GT: Tonelaje de arqueo bruto (grosstonage). Capturas en kg. }\end{array}$} \\
\hline & Embarcaciones & GT & Capturas \\
\hline Arrastre & 243 & $13.491,70$ & 8.473 .212 \\
\hline Artes menores & 404 & $1.674,10$ & 1.770 .936 \\
\hline Cerco & 84 & $3.211,40$ & 16.506 .319 \\
\hline Cerco atunero & 6 & $1.612,40$ & 0 \\
\hline Palangre de fondo & 42 & 413,2 & 225.920 \\
\hline Palangre de superficie & 13 & 389,9 & 372.957 \\
\hline Auxiliares & 45 & 405 & 0 \\
\hline Mariscadores & 0 & 0 & 316.631 \\
\hline Total & 837 & $21.197,80$ & 27.665.975 \\
\hline
\end{tabular}

Fuente: Instituto de Estadística de Cataluña. ${ }^{4}$

español. ${ }^{3}$ En este marco estatal, la flota en Andalucía prácticamente dobla a la de Cataluńa, y la de Galicia es cinco veces mayor. La explotación de los recursos pesqueros en Cataluña se limita básicamente a la pesca litoral, desde la misma línea de costa hasta profundidades próximas a los mil metros. A lo largo del año, en las lonjas de pescado de Cataluña se comercializan unas 120 especies diferentes.

Como se ve, la pesca catalana, aunque no muy rica, es muy diversa, lo cual se traduce también en una gran variedad culinaria. Las especies más habitualmente capturadas son las siguientes; de pescado azul: sardina, boquerón, jurel, caballa, bonito, etcétera; de pescado blanco: besugo, congrio, lenguado, merluza, mero, dorada, rape, entre otros. Los cefalópodos más pescados son el pulpo de roca, la sepia y el calamar; los crustáceos, la gamba, la cigala, el langostino y la langosta; y finalmente entre los moluscos cabe destacar el cultivo del mejillón en la zona del delta del Ebro, además de la almeja, berberecho, la navaja, la ostra, etcétera, aunque es una pesca muy en declive a causa de la erosión del lecho marino. Cabe destacar la captura y engorde del atún rojo, aunque es pescado básicamente por una única empresa situada en L'Ametlla de Mar (Tarragona) que representa solo el $0,7 \%$ de la flota catalana, $y$ en cambio estas seis embarcaciones son responsables

3 Instituto Nacional de Estadística http://www.ine.es/ prensa/cre_2016_1.pdf Consultado 3.01.2017. ellas solas del 7,6\% del total del GT de pescado capturado, como puede observarse en la Tabla 1.

Conviene recordar, aunque pueda resultar evidente al lector, que las poblaciones de pescadores se han visto afectadas, a través del tiempo, por fuerzas que iban mucho más allá de su contexto inmediato. Para entender el contexto en que desarrolla la pesca en Cataluña en la actualidad, debemos situarlo en relación a la principal actividad productiva de las costas, que no es la pesca sino el turismo. Según la Organización Mundial del Turismo, ${ }^{5}$ España es el tercer destino turístico mundial después de Estados Unidos y Francia. Cataluña, y concretamente la costa catalana, es el principal destino turístico de España, tanto en número de turistas como en ingresos globales (Jiménez y Prats, 2006).

Cataluña tiene una población de 7,5 millones de habitantes y recibe anualmente alrededor de 17 millones de turistas, cifra que representa cerca del $2 \%$ del total mundial (Jiménez y Prats, 2006). Aproximadamente el $45 \%$ del turismo se concentra en las pequeńas y medianas poblaciones de costa y otro 50\% en la ciudad de Barcelona (Generalitat de Catalunya, 2013, p. 26). Solamente en la Costa Brava (Girona),

4 http://www.idescat.cat/pub/?id=aec $\&$ n= 468\&lang=es Consultado 28.12.2016.

5 http://mkt.unwto.org/sites/all/files/docpdf/unwtohighlights11enlr_3.pdf Consultado 11.02.2017. 
con 665 mil habitantes permanentes, acuden anualmente 5,5 millones de turistas. Una parte importante de ellos son residenciales, lo que ha comportado un fuerte impacto en el crecimiento urbanístico de las últimas décadas. En la Costa Brava, de acuerdo con los datos que aporta Saló (2006) en su tesis doctoral, la construcción de segundas residencias llegó a triplicar, en los años noventa, la construcción de viviendas destinadas a residencia habitual. De acuerdo con estimaciones del Instituto Catalán de Estadística, ${ }^{6}$ en los municipios costeros la población estacional supera en más de un $40 \%$ a la población residente, lo que tiene un claro impacto ambiental en aspectos como el consumo de agua o la gestión de los residuos. Según la Red de Seguimiento Ibérico del Litoral Marino (Proyecto Silmar), ${ }^{7}$ entidad que a través de diferentes estaciones de control se encarga de analizar la evolución del estado ecológico del fondo marino y la costa, en la costa catalana, y particularmente en la Costa Brava (Girona) y la Costa Dorada (Tarragona), el fondeo masivo de embarcaciones deportivas es, junto a otros factores derivados de la masificación urbana como la contaminación debida a la sobrepoblación durante el verano y la construcción de infraestructuras turísticas, la principal causa de degradación de los ecosistemas marinos.

La crisis de la pesca, y en concreto del sector pesquero artesanal a escala mundial, lleva décadas anunciándose (McGoodwin, 1990; Crean y Symes, 1996), así como también lleva tiempo anunciándose a escala local de las comunidades de pescadores en España (Freire y García-Allut, 2000; Tudela, 2002; Florido, 2004; Beorlegui y Sotes, 2009; Varela y Ojeda, 2010). Todos los expertos coinciden en señalar como causantes de esta crisis pesquera -aparte de la contaminación- la sobreexplotación motivada por el desarrollo técnico y la expansión hacia nuevos ecosistemas marinos. A ello hay que añadir a nivel local español en los últimos años la competencia y descenso en los precios del pescado, al mismo tiempo que el aumento de los costes por la crisis del petróleo y otras inversiones derivadas de la necesidad de adaptar

6 Ver: http://www.diaridegirona.cat/temadia/2012/04/14/poblacio-estacional-supera-lempa dronada-al-70-municipis-majors-5000-habitants/557101. html Consultado 11.02.2017.

7 http://fundacionmar.org/ca/projecte-silmar/1-projectesilmar/ Consultado 11.02.2017. la flota a normativas cada vez más exigentes. Así por ejemplo, la Confederación Española de Pesca advertía por carta al gobierno español de la grave amenaza que se cernía sobre el sector cuando el precio del carburante había aumentado entre 2008 y 2012 un $40 \%$, mientras que el precio de ciertas especies como el jurel o la merluza había llegado a bajar en las lonjas españolas más del $50 \%$ en el mismo lapso de años. ${ }^{8}$

Las cifras de la flota pesquera catalana en la actualidad nos muestran una profunda crisis que no se distribuye por igual sino que afecta fundamentalmente a la flota artesanal (Lleonart, 1996; Alegret y Garrido, 2008; Böcker, 2013). Si observamos la evolución de la flota catalana por modalidades (Tabla 2), veremos que entre 2003 y 2015 se ha reducido en un $40 \%$. Este descenso afecta principalmente a la flota artesanal, ya que las embarcaciones de artes menores se han visto reducidas en el mismo período en prácticamente un 50\%, mientras que, por ejemplo, las embarcaciones de cerco lo hacían en un 30\%. Para ampliar esta información, en la Figura 1 podemos observar la evolución descendente de toneladas de pescado capturadas en Cataluña, así como la evolución de la recaudación económi$\mathrm{ca}$, igualmente en un pronunciado descenso si nos fijamos en la recaudación constante, es decir, aquella cifra corregida por la evolución del IPC, que se ha reducido prácticamente a la mitad en el lapso de años (1981-2017) que abraza el gráfico elaborado por el Departamento de Agricultura, Ganadería, Pesca y Alimentación. Esta visión panorámica nos permite situar en cifras la crisis que está viviendo el sector pesquero en Cataluña donde, según datos de Greenpeace (2013), en tan solo cinco años se han destruido un tercio de los puestos de trabajo. Si tomamos los datos oficiales del gobierno español, en el conjunto de España el número de empleados en la pesca y acuicultura se ha reducido un $33 \%$ entre 2002 y 2015. ${ }^{9}$ Nos encontramos pues ante un

8 Redacción. "Gasoil y precios en origen ponen a la flota en situación límite", Industrias pesqueras.com (30 de enero de 2012). Ver: http://www.industriaspesqueras. com/noticias/en_portada/19840/gasoil_y_precios_en_ origen_ponen_a_la_flota_en_situacion_limite.html Consultado 3.06.2017.

9 http://www.mapama.gob.es/es/estadistica/temas/estadisticas-pesqueras/2015_01_trabajadores_afiliados_ tcm7-195611.pdf Consultado 11.02.2017. 
Tabla 2. Evolución de la flota pesquera en Cataluña.

\begin{tabular}{|c|c|c|c|c|c|c|c|c|c|c|c|c|c|}
\hline \multirow[t]{2}{*}{ Modalidad } & \multicolumn{13}{|c|}{ Número embarcaciones } \\
\hline & 2015 & 2014 & 2013 & 2012 & 2011 & 2010 & 2009 & 2008 & 2007 & 2006 & 2005 & 2004 & 2003 \\
\hline Arrastre & 243 & 256 & 272 & 271 & 285 & 288 & 298 & 309 & 331 & 346 & 346 & 349 & 356 \\
\hline Artes menores & 404 & 422 & 447 & 469 & 553 & 549 & 537 & 598 & 700 & 771 & 779 & 787 & 796 \\
\hline Atún (cerco) & 6 & 6 & 6 & 6 & 6 & 6 & 6 & 6 & 6 & 6 & 6 & 6 & 6 \\
\hline Cerco & 84 & 86 & 87 & 88 & 85 & 91 & 93 & 95 & 100 & 109 & 112 & 113 & 119 \\
\hline Palangre de fondo & 42 & 45 & 45 & 46 & 45 & 54 & 53 & 52 & 56 & 68 & 68 & 72 & 72 \\
\hline $\begin{array}{l}\text { Palangre de } \\
\text { superficie }\end{array}$ & 13 & 13 & 12 & 11 & 12 & 12 & 12 & 10 & 10 & 11 & 10 & 10 & 9 \\
\hline Auxiliares & 45 & 41 & 46 & 46 & 6 & 39 & 37 & 44 & 43 & 38 & 30 & 24 & 24 \\
\hline Total embarcaciones & 837 & 869 & 915 & 937 & 1.022 & 1.039 & 1.036 & 1.114 & 1.246 & 1.349 & 1.351 & 1.361 & 1.382 \\
\hline
\end{tabular}

Fuente: Departamento de Agricultura, Ganadería, Pesca y Alimentación. Gobierno de Cataluña. ${ }^{10}$

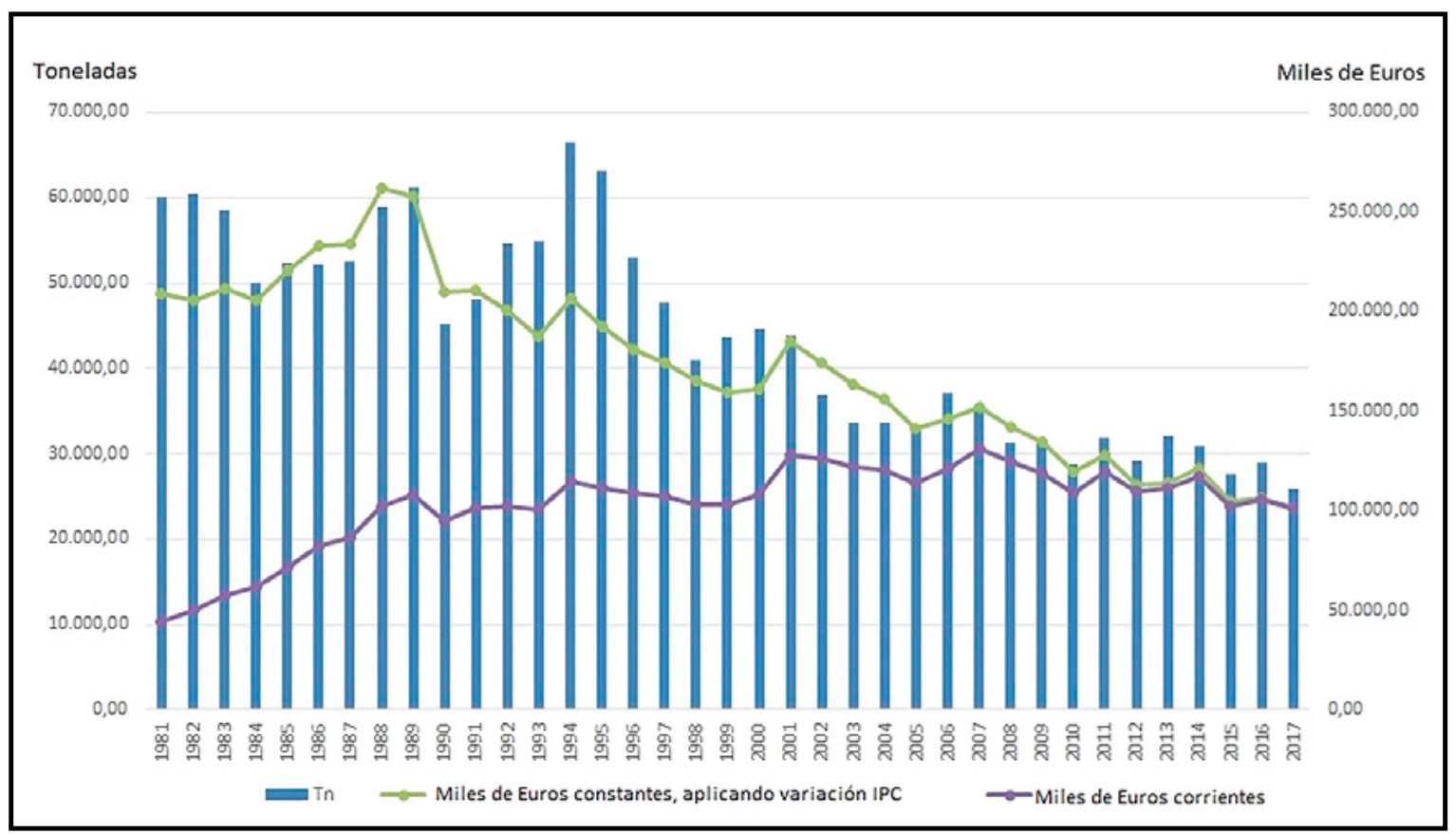

Figura 1. Evolución de las toneladas de capturas y la recaudación económica de la pesca en Cataluña. Fuente: Departamento de Agricultura, Ganadería, Pesca y Alimentación. Gobierno de Cataluña. ${ }^{11}$

panorama de desempleo ciertamente preocupante, incluso nos atreveríamos a calificar de angustiante para muchos pescadores artesanales, algunos de ellos

10 http://agricultura.gencat.cat/ca/ambits/pesca/dar_flota_pesquera/dar_estadistiques/dar_situacio_flota_pesquera/ Consultado 28.12.2016. nietos y bisnietos de pescadores, en unas costas que para muchas personas del mundo son sinónimo de vacaciones, buena comida, buen clima y diversión.

11 http://agricultura.gencat.cat/ca/ambits/pesca/dar_estadistiques_pesca_subhastada/dar_subhasta_llotges_catalanes/ Consultado 7.04.2018. 


\section{La patrimonialización del ámbito marítimo-pesquero en Cataluña}

La desaparición de la mitad de las embarcaciones de pesca artesanal (desaparecieron unas 400 embarcaciones de artes menores, la mitad del total, en 12 años, como puede verse en la Tabla 2) contrasta con un movimiento social, impulsado por clases medias, por los habitantes de poblaciones costeras pequeñas y medianas, para la recuperación de embarcaciones de madera destinadas a la navegación a vela tradicional socializada y en grupo, no privada o particular, ${ }^{12}$ y en general para proteger y conservar los últimos vestigios del patrimonio marítimo que no han sucumbido a la presión urbanística en el litoral: inmuebles relacionados con la pesca artesanal, como pequeñas atarazanas, almacenes, locales donde teñir las redes, casetas con maquinaria de remolque, estaciones de salvamento marítimo, faros, etcétera (Carbonell y Domènech, 2017). Algunos de estos edificios han sido reconvertidos en los últimos años en pequeños museos y centros de interpretación, bajo el impulso de asociaciones culturales y poderes locales, básicamente ayuntamientos, con financiación también en principio local, aunque se haya podido contar en ocasiones con subvenciones del gobierno autonómico y, excepcionalmente, de alguna empresa privada. En su mayor parte este movimiento de salvaguarda del patrimonio marítimo ha sido impulsado por asociaciones culturales creadas con tal fin. Diez de estas asociaciones están agrupadas en la "Federació catalana per la cultura i el patrimoni marítim i fluvial", ${ }^{13}$ aunque existen otras asociaciones no federadas. A pesar de que alguna de ellas se fundó a mediados de los años ochenta, la mayoría de las asociaciones fueron creadas con posterioridad al año dos mil.

El surgimiento de estas asociaciones puede explicarse, en parte, por la importancia y vitalidad del tejido asociativo catalán de carácter cultural y festivo, articulado en torno a docenas de asociaciones de ámbito local. Estudiando la cultura festiva en Cataluña, dos antropólogas estadounidenses han mostrado,

12 He trabajado esta cuestión en otro lugar. Ver Carbonell 2014 y 2015.

13 Ver http://fccpmf.blogspot.com Consultado 22.05.2017. en sendos trabajos (Kammerer, 2014; Vaczi, 2016), que se trata de un asociacionismo con características singulares, muy vinculado a la reivindicación nacionalista de la identidad social y cultural catalana. En un plano más general, este asociacionismo cultural y festivo también se puede vincular a la voluntad, tan a menudo expresada a través del patrimonio cultural, de reafirmar la cultura local frente a la globalización (Prats, 1997; Frigolé y Roigé, 2006). Pero por otra parte, finalmente, también se puede relacionar con una creciente demanda de turismo cultural en áreas costeras, como por ejemplo el llamado "pescaturismo" o turismo pesquero, que a falta de una regulación española al respecto, como la que existe en Italia desde 1999, algunos gobiernos autonómicos, Cataluña entre ellos, han empezado, en el ámbito de sus competencias, a regular y potenciar (Santana y Pascual, 2003; Pascual, 2004; Gracia, 2016; Palou, 2017). En general se observa la llegada a las costas mediterráneas de nuevos turistas ávidos por conocer lo que MacCannell (1976) denominó el "back" del escenario turístico, como son por ejemplo los puertos y espacios de trabajo de los pescadores.

Lo cierto es que en las mismas playas donde hace dos o tres décadas el patrimonio marítimo yacía abandonado, hoy podemos contemplar una interesante dinámica de conservación y puesta en valor del mismo patrimonio. Por ejemplo, en Sant Pol de Mar (Barcelona), un pueblo cuya economía se había basado largamente en la pesca y la agricultura, en diciembre de 1990 se incineraba sobre la playa la última barca de "bou", una gran barca de pesca artesanal de madera. Las autoridades consideraron que la embarcación abandonada por su armador constituía un estorbo y un peligro para los turistas. Nadie propuso salvarla. En el mismo lugar, en el año 2001, nacía "A tot drap" (A todo trapo), una asociación para la protección de lo que quedaba del patrimonio marítimo local, movilizando un grupo importante de personas, que invirtieron tiempo y esfuerzos en restaurar embarcaciones, edificios y útiles de pesca. ${ }^{14}$ Lo mismo acontecía en otras poblaciones de la costa contemporáneamente. Ello fue lo que nos llevó a un grupo de antropólogos a impulsar un proyecto de investigación para conocer con exactitud el alcance

14 He descrito con más detalle este caso en otro lugar, ver Carbonell (2013). 
del movimiento que se estaba generando en torno al patrimonio marítimo.

Entre los ańos 2011 y 2013 llevamos a cabo un sondeo en los 64 municipios que hay a lo largo de los $580 \mathrm{~km}$ de costa catalana, con el fin de recolectar datos concretos sobre las acciones que se estaban llevando a cabo en torno al patrimonio marítimo material e inmaterial. ${ }^{15}$ Se registró en una base de datos un total de 773 acciones de patrimonialización en Cataluña durante esos tres años, que comprendían desde pequeños actos festivos hasta intervenciones arquitectónicas, todas las acciones que se llevaban a cabo en torno al patrimonio marítimo en cada una de las poblaciones costeras. El estudio permitió obtener los siguientes datos generales: El mayor número de acciones tenía lugar en las poblaciones con mayor importancia pesquera, como Palamós, Vilanova o Sant Carles de la Ràpita. En cuanto a la tipología de acciones, destacaban aquellas relacionadas con el patrimonio inmaterial (52\% del total), seguidas del patrimonio mueble (33\%), incluyendo las embarcaciones tradicionales, y en menor medida las acciones sobre el patrimonio inmueble (8\%) y patrimonio natural $(7 \%)$.

La preponderancia de las acciones de patrimonio inmaterial se explica, por una parte, por su coste

15 Proyecto I+D+I PESCUM impulsado desde la Universitat de Girona y financiado por el Ministerio español de Ciencia e Innovación (Ref. HAR2010-15566). Se realizó una base de datos en colaboración entre el Institut Català de Recerca en Patrimoni Cultural y el Centre de Documentació Marítima del Museu de la Pesca de Palamós. La metodología consistió en consultas telefónicas a todos los ayuntamientos, rastreo de páginas web, Facebook y blogs de asociaciones y entrevista a informantes destacados. En la BBDD se registraron los siguientes datos sobre las acciones de patrimonialización: nombre de la acción, descripción, localización, fechas, periodicidad, afluencia estimada, precio, contexto festivo, promotores, fuentes de financiación, y a continuación una serie de categorías y subcategorías para clasificar el ámbito del patrimonio marítimo sobre el que se actuaba, ya fuera patrimonio mueble, inmueble, inmaterial o natural. El proyecto incluía un estudio comparativo entre Cataluña, País Vasco, Galicia y Andalucía, aunque en este artículo solamente presentamos los datos relativos a Cataluña. Ver http://icrpc.cat/es/ proyectos/patrimonio-etnologico-sociedad-y-culturamaritima-pescum.html Consultado 26.05.2017. económico, generalmente menor en comparación con la intervención sobre el patrimonio mueble e inmueble. Pero por otra parte, este predominio del patrimonio inmaterial se puede explicar también por su relación con la explotación del patrimonio marítimo desde la perspectiva del turismo. La mayoría de las acciones en el ámbito del patrimonio inmaterial consistían en actividades con una clara vocación turística, llevadas a cabo en el marco de las fiestas mayores que tienen lugar en las poblaciones de costa. Cabe destacar las ferias gastronómicas, ya que prácticamente todas las poblaciones costeras organizan a lo largo del año algún tipo de evento centrado en productos pesqueros o recetas marineras: jornadas gastronómicas del pulpo, del calamar, del atún, del langostino, de la cigala, de los erizos de mar, etcétera. Se trata de actividades muy enfocadas a la promoción económica del sector restauración y comercial. También hay que incluir aquí los festivales de música marinera y los mercados artesanales y ferias de oficios donde se reproducen técnicas y saberes antiguos vinculados a la pesca. En este último aspecto se incluían una gran variedad de actividades, como las recreaciones históricas, con atrezo e indumentaria de época, demostraciones de sistemas de pesca artesanal en desuso, demostraciones de subasta pública de pescado, demostraciones y talleres didácticos de oficios marineros organizados por los museos locales, etcétera. Todas estas actividades iban destinadas en gran medida al consumo turístico mayormente doméstico, pero también extranjero.

Hay de todos modos otro ámbito importante del patrimonio inmaterial no destinado específicamente al turismo. Es el que tiene que ver con la recuperación de embarcaciones tradicionales, muy activo por parte de asociaciones locales, y las campañas populares para la salvaguarda de edificios, maquinaria o infraestructura en desuso. Durante el período estudiado (2011-2013), recogimos 25 acciones de recuperación de embarcaciones tradicionales y 45 acciones de puesta en valor de estas embarcaciones, como encuentros y navegaciones conjuntas, demostraciones técnicas como la botadura desde la playa, desembarcos y recreaciones de hechos relevantes de la historia de Cataluña. En cuanto al patrimonio inmueble, en el mismo período registramos 15 intervenciones arquitectónicas de restauración y 67 acciones de puesta en valor del patrimonio inmueble, 
como su señalización, inclusión en rutas autoguiadas, visitas guiadas, publicaciones, exposiciones, entre otras. Estas acciones, llevadas a cabo no necesaria o exclusivamente para el turismo, eran en su mayoríae promovidas desde las entidades locales surgidas de movimientos asociativos a los que me he referido antes.

Analizando quienes eran los promotores de todas las acciones de patrimonialización, concluimos que la mayoría, el 36\%, estaban organizadas por asociaciones locales, seguidas por este orden de ayuntamientos, museos y empresas privadas. Estas asociaciones culturales están formadas por voluntarios, personas interesadas por el patrimonio marítimo y la navegación tradicional, muchos más hombres que mujeres, generalmente de mediana edad, incluyendo a personas de muy diversas profesiones, desde empleados a profesionales liberales, también algunos pescadores activos o personas descendientes de familias de pescadores que abandonaron el oficio pero que mantienen una relación sentimental o nostálgica con la pesca. Como veremos en el siguiente apartado, algunos pescadores están aprovechando lo que Jane Nadel-Klein (2003) en su libro Fishing for heritage denominó el "boom patrimonial" en la pesca para reconvertir su actividad en crisis. La pregunta que nos podemos hacer es si finalmente se está produciendo una sustitución de la pesca por el turismo a través del patrimonio.

\section{Dos testimonios}

Con el fin de ejemplificar y ver más de cerca el proceso que está aconteciendo actualmente en el ámbito de la pesca artesanal en Cataluña, a continuación presento dos testimonios que me parecen significativos. Ramon y Josep son dos pescadores del puerto de Arenys de Mar (población de 15 mil habitantes situada a $40 \mathrm{~km}$ al norte de la ciudad de Barcelona) que durante una etnografía que realicé de dicho puerto entre los años 2009 y 2010 fueron dos de mis principales informantes (Carbonell, Doñate y Romero, 2011). En aquel momento ambos pertenecían ya a sendas asociaciones culturales dedicadas al patrimonio marítimo, las cuales habían ayudado a formar y que incluso presidieron durante unos años. Era aquella una actividad a la que dedicaban su tiempo de ocio, ya que además de pescadores profesionales son dos personas muy apegadas a la cultura marítima. Ramon formaba parte de la asociación "Grup Llebeig” (nombre catalán del viento $\mathrm{SW}$ ) que participaba en fiestas y ferias de los pueblos vecinos para promover la cultura marítima. Un ejemplo de ello eran las demostraciones de subasta pública de pescado en la playa o en las plazas, como se hacía antes de que desde la administración se obligara a trasladar las subastas al interior de las lonjas de pescado, cerrando el acceso a los no profesionales. Durante las subastas, en forma de espectáculo, que organizaba esta asociación con motivo de fiestas y ferias locales el público asistente, además de adquirir pescado, escuchaba las explicaciones que les daba Ramon sobre las cualidades gastronómicas y formas de cocinar algunas especies poco consumidas en los hogares hoy en día. Por su parte, Josep fundó también con un grupo de amigos, amantes de la navegación tradicional, la asociación "A tot drap", antes citada, con el fin de restaurar embarcaciones de madera y dotarlas con aparejo de vela latina, así como también participar en fiestas y ferias en distintos pueblos de Cataluña, realizando demostraciones de botadura desde la playa, pesca artesanal, navegaciones conjuntas, etcétera.

Ramon y Josep llevan la pesca en las venas, como se suele decir. Son pescadores de profesión y de vocación. Así y todo, no han podido resistir la crisis del sector y finalmente han optado por vender su barca de pesca e ir a otra cosa. A través de su testimonio podemos ver las estrategias que han desarrollado ambos para mantenerse vinculados al universo marítimo-pesquero, reciclando sus saberes hacia actividades de cariz patrimonial, rentabilizando su capital cultural para poder subsistir sin tener que alejarse totalmente de lo que, como hemos visto, más que un trabajo es para ellos un modo de vida, una forma de estar en el mundo. En diciembre de 2016 realicé una larga entrevista semiestructurada a cada uno de ellos por separado, tomando como base la historia de vida, que paso a resumir a continuación con el fin de ejemplificar un proceso que se está viviendo actualmente en la pesca en Cataluña. 


\section{Ramon: Del trasmallo al grill}

Ramon Tarridas ha sido pescador artesanal durante cerca de 30 años en el puerto de Arenys de Mar junto con su hermano. Los hermanos Tarridas son la quinta y, muy probablemente, la última generación de pescadores en su familia, dedicada a la pesca con redes de trasmallo. Además de las barcas de pesca, la familia posee los "Baños Tarridas", un pequeño negocio de temporada, que se monta y desmonta cada verano sobre la playa, consistente en unas casetas de baño y un pequeño bar, del que tienen constancia documental desde el año 1913, aunque opinan que puede ser más antiguo. Como he dicho antes, además de pescador Ramon es un hombre comprometido con la cultura marítima y la pesca artesanal. En este sentido, en el año 2006 asumió la presidencia de la "Asociación de Armadores de Artes Menores de Cataluña”. Coincidió que en aquel período se estaba discutiendo en el Parlamento Europeo la llamada Política Pesquera Común (PPC). Algunas ONG como WWF y Accionatura se ponen en contacto con él y durante tres ańos le invitan a participar en debates y comisiones internacionales para hacer oír la voz de los pescadores artesanales de Europa: "Yo creo que este fue mi mal. Porque tengo una visión global y veo que para solucionar el problema debe haber una decisión politica muy valiente y no veo esta valentía. Y lo digo con tristeza. No veía esta valentía. Ni la veo en mis compañeros pescadores. La gente tiene su mundo, su barca, su calador, su casa, pero no la marees con nada más". ${ }^{16}$ En verano de 2015 dejó de pescar y en otoño del mismo año abrió con su mujer y sus hijas un restaurante dedicado al pescado de proximidad cocinado al grill.

Ramon comenzó a pescar con su padre y su tío en el año 1987. Era una época en que la pesca iba en aumento hasta tocar techo hacia el ańo 2000. En el arte del trasmallo, las redes habían evolucionado, los motores de las embarcaciones, la tecnología, todo ello permitía pescar más lejos y a más profundidad. En sus palabras: "habias ganado mar". Ramon ve la evolución de la pesca que él ha vivido en una serie de revoluciones, de las cuales queda la más trascendente por hacer:

16 Todos los fragmentos en itálicas de este apartado son transcripciones literales de entrevista.
En el año 1975 llega la revolución de la dimensión. Se hacen barcas más grandes. Hacia 1990 llega la revolución de mayores potencias de motores. Y después, ya llega el año 2000, la revolución tecnológica. Tenemos entonces aqui un coctel, pensando siempre, como los pescadores pensábamos, que el pescado era infinito. $Y$ que a pesar de que aumentaban la eslora de las barcas, la potencia, la tecnología, los peces no se acabarian nunca. Y lo que todavía no ha llegado, que espero que llegue pronto, es la revolución de la gestión. Es la que falta. No puedo hablar por toda España y todo el mundo. Hablo por el Maresme, por Arenys, la zona que conozco más profundamente, y podría decir Cataluña, lo que no se ha aplicado todavía es la gestión. Tenemos pequeños modelos de gestión que conocemos, el tema del sonso, el tema del atún, los dos por necesidad, porque la especie habia llegado a punto del colapso. La solución fue aplicar modelos de cogestión.

La temporada de verano de 2011 Ramon y su hermano toman el relevo generacional en el negocio de la playa, los "Bańos Tarridas", que manejaban sus padres y sus tíos. Deciden introducir un nuevo concepto de cocina basado en el pescado de proximidad, "no de temporada sino del dia", precisa Ramon. Cada día el menú se establece en función de lo que trae la barca de pesca y se dan a conocer las cualidades gastronómicas de los peces de bajo precio en la lonja. El modelo les funcionó muy bien los siguientes veranos $y$, ante los casi nulos rendimientos de la barca de pesca, en 2015 deciden poner en venta sus dos embarcaciones. El hermano de Ramon abre con su mujer un negocio de comida casera para llevar, y Ramon abre también con su mujer y sus hijas un restaurante dedicado al pescado cocinado a la parrilla, siguiendo el mismo principio de ofrecer pescado del día y de bajo precio. Además del comedor, el restaurante dispone de una sala donde ofrecen "showcookings", cursos de cocina de pescado, talleres extraescolares para niños de 6 a 12 ańos, charlas sobre pesca artesanal y consumo responsable, en sintonía con lo que están haciendo algunas ONG. ${ }^{17}$ Aunque

17 http://www.wwf.es/que_hacemos/mares_y_costas/ nuestrs_soluciones/pesca_sostenible/fish_forward/ 
Ramon ha decidido que no volverá a pescar, tiene muy claro que su trabajo actual está relacionado con su oficio anterior: "Porque yo, al final, sigo viviendo de mis conocimientos. Antes los ponía en práctica para ir a pescar y ahora los pongo en práctica cocinando y haciendo divulgación de mis conocimientos".

\section{Josep: Del palangre al pailebote}

A diferencia de muchos de sus compañeros, Josep no proviene de una larga estirpe de pescadores artesanales; de hecho no tenía ningún pariente pescador y sus padres fueron los primeros sorprendidos cuando les anunció que iba a ser pescador. Nacido en Arenys de Mar, desde muy joven se sentía atraído por los deportes marítimos, así que al llegar el momento de buscarse un trabajo no lo pensó dos veces. Al regresar del servicio militar, en el ańo 1982, para un joven como Josep la pesca profesional ofrecía un buen salario, de los mejores que podían encontrarse para trabajadores sin formación universitaria. Es así que decide embarcarse en un barco de arrastre, el Ictíneo, donde irá aprendiendo el oficio junto a otros ocho tripulantes. En 1985 España firma el tratado de adhesión a la Unión Europea. Josep recuerda que al poco tiempo empezó a llegar ayuda financiera de la UE para modernizar la flota pesquera. Se invirtió en motores más potentes, en tecnología de pesca, como la sonda y el LORAN, ${ }^{18}$ en artes de pesca más eficaces. En los años noventa hubo, en sus palabras, "un boom de la pesca". Mientras conversamos en un café cerca del puerto de Arenys de Mar, señala a un anciano en una mesa cercana:

Aquel hombre iba con el reloj y el compás. Con el LORAN las rocas fueron súper explotadas. Porque antes, cuando estabas fuera y no veías las señas de tierra, las rocas no siempre se encontraban. Y entonces se empezó a hacer daño, con todos esos aparatos se fue agotando el mar. También reconoce el daño del arrastre: el arrastre acabó por ser como arar un campo, vas arando el barro y los peces han desaparecido.

nueva_guia_de_consumo_de_pescado_wwf/ Consultado 5.01.2017.

18 LORAN: LOng RAnge Navigation, sistema de ayuda a la navegación anterior al GPS.
Durante muchos años pescó merluza a palangre con su barco Tramontana, que tenía a medias con otro socio. También tuvo durante unos años un pequeño arrastre con el mismo socio. A finales de los años noventa y comienzos de la década siguiente es cuando empieza a darse cuenta de que la pesca va cada ańo peor. Los beneficios se reducen porque disminuyen progresivamente las capturas y el precio del pescado. A la sobreexplotación ańade dos razones más. Por una parte la contaminación del mar. Se había encontrado en ocasiones con que al levar las redes el $80 \%$ del contenido eran plásticos y desechos: "Siempre ves la huella humana, cales por donde cales, siempre hay huella humana". La otra razón fue la profusión de supermercados que vendían pescado de importación, a la vez que iban cerrando las pescaderías locales y acudían menos compradores a la subasta de la lonja del puerto. A partir de 2007, con la llegada de la crisis financiera global y, en Espańa particularmente, a partir del 2008 con la crisis del mercado inmobiliario y el creciente desempleo hasta alcanzar el 25\% en 2012, la pesca siguió siendo un oficio afectado por los salarios decrecientes en el conjunto la crisis de todos los sectores productivos. Esta situación afectó negativamente al consumo del pescado, sobre todo el más caro como la merluza que pescaba Josep con el palangre de fondo. En cambio la sardina, que ellos usaban de cebo, al ser un pescado más barato, aumentó la demanda y el precio. La situación se fue haciendo cada vez más insoportable. Al final, de los cinco días laborables de la semana, cuatro eran para cubrir gastos y solamente uno permitían beneficios para repartir entre los dos socios de la embarcación. En invierno, cuando llegaban días de mal tiempo en que no se podía salir a pescar, las pérdidas eran insostenibles y tuvieron que recurrir a pólizas de crédito:"era una pesadilla".

Josep siempre ha tenido, como dice él mismo, "una vida paralela a la de pescador". Los días libres los dedicaba a navegar en embarcaciones tradicionales. Como hemos visto antes, en Cataluña en los años dos mil hubo una especie de despertar del patrimonio marítimo, que se tradujo entre otras cosas en el nacimiento de un gran interés por la navegación con embarcaciones tradicionales a vela latina. Josep, como dijimos, fundó con otros compañeros la asociación "A tot drap" y además colaboró durante estos años 
de una forma muy activa con asociaciones de distintas poblaciones de toda la costa catalana, llegando a ser una persona muy conocida en este ambiente. En el 2016, el Museo Marítimo de Barcelona sacó a concurso la gestión del pailebote Santa Eulàlia, un velero histórico declarado Bien Cultural de Interés Nacional (BCIN) que forma parte de la flota histórica del museo. La empresa armadora que ganó el concurso propuso a Josep como patrón segundo o contramaestre del velero histórico: "Creo que me embarqué un miércoles y el martes estaba todavía pescando. Fue como un hachazo. El día 1 de junio de 2016. Que por cierto estoy muy bien. Me queda un año y medio para poder jubilarme, pero si sigue asi yo no me jubilo, yo no me veo en tierra jubilado". Toda una declaración de intenciones.

\section{Reflexiones finales}

En este artículo he tratado de proyectar una mirada antropológica a la situación actual de la pesca artesanal después de haber estudiado de cerca la patrimonialización de la pesca en la costa catalana, y en especial en la costa norte de Barcelona, durante los últimos 10 ańos. Inicialmente me pareció ver en el patrimonio una oportunidad para la pesca artesanal, ya que algunos de mis informantes pescadores participaban del movimiento de recuperación del patrimonio marítimo que se extendió por la costa durante la primera década del siglo XXI. Ahora, en cambio, tiendo a preguntarme si la patrimonialización no habrá sido una herramienta más en un proceso que empezó a substituir pescadores por turistas en las costas mediterráneas hace ya más de medio siglo. He tomado en cuenta también la dimensión histórica para dejar abierta la pregunta de Perejaume relativa a saber si aquel pescador que se ha involucrado en la esfera del turismo volverá tal vez algún día a ser pescador. La historia nos muestra que la pesca en Cataluña ha seguido un ritmo oscilante y que el pescador catalán históricamente se ha visto obligado a combinar su actividad en el mar con ocupaciones terrestres para poder sobrevivir en un medio poco generoso en recursos pesqueros.

Con la revolución tecnológica llegó a ser incluso una ocupación atractiva para jóvenes a quienes les gustara el oficio, que ofrecía no solo buenas ganancias sino también una vida en contacto con el mar
Mediterráneo, cuyas condiciones de trabajo no son ni mucho menos comparables con la dureza en ciertas latitudes del mar abierto donde la pesca es una actividad ciertamente arriesgada. Pero la revolución tecnológica en la pesca llevó a la sobreexplotación del recurso, cosa que, sumada a la degradación ambiental provocada por la sobrepoblación estacional en las costas derivada del turismo de masas, llevaría en pocos años a la crisis pesquera en la que nos encontramos actualmente. Hay que añadir aquí el contexto de crisis económica generalizada en la que se han encontrado los trabajadores españoles en los últimos años, con una tasa de desempleo por encima del $20 \%$ durante 66 meses seguidos entre 2010 y 2015 , llegando al $26,94 \%$ en el punto máximo. ${ }^{19}$ En esta situación, solamente algunos pescadores disponen del capital, ya sea monetario o en términos bourdieanos de capital social y capital cultural, necesario para dar el salto a otras ocupaciones.

En el caso de Ramon, ha debido recurrir a un crédito bancario para empezar el negocio del restaurante al grill. El producto gastronómico que ofrece en su establecimiento trata de distinguirse en un mercado tan competitivo como es la restauración, poniendo en juego sus conocimientos sobre la pesca sostenible para atraer a los "ethical consumers", es decir, aquellos consumidores que se preocupan por la procedencia del producto (Newholm y Shaw, 2007, p. 255) y que están también vinculados a otro nuevo concepto como es el de "turismo responsable" (Gascón y Cañada, 2005). Su experiencia no solo como pescador sino también como activista y representante de los pescadores artesanales ante el Parlamento Europeo, en contacto durante años con ONG ambientalistas y los biólogos marinos, le han servido de capital cultural para construir su alternativa personal de las redes de trasmallo al restaurante al grill de pescado de proximidad.

En el caso de Josep, han sido sus relaciones tejidas durante ańos con los sujetos de distintas asociaciones culturales dedicadas a la conservación del patrimonio marítimo local las que constituyen su capital social

19 Ver diario El País: "España sufre su periodo más largo con el paro por encima del 20\%”. http://economia.elpais.com/economia/2016/04/28/actualidad/1461866236_955613.html Consultado 8.06.2017. 
que le ha permitido acceder a un nuevo puesto de trabajo relacionado con el mar. Han sido varios años acumulando experiencia en embarcaciones históricas, navegando con diferentes personas, participando en encuentros de embarcaciones realizados de levante a poniente de la costa catalana, tripulando embarcaciones históricas a vela, grandes y pequeñas, como ha ido acumulando también el capital cultural necesario para optar al cargo de contramaestre del Santa Eulàlia. Todo esto no habría tenido lugar sin el boom patrimonial que se ha vivido en Cataluña en el ámbito marítimo en los últimos 15 años. El pailebote Santa Eulàlia, una goleta de tres palos construida en 1918, adquirido por el Museo Marítimo de Barcelona en 1997 y declarado Bien Cultural de Interés Nacional en 2011, es seguramente la más importante de las embarcaciones históricas en $\mathrm{Ca}$ taluña. Ocupar un cargo relevante en la tripulación no es para Josep un oficio como otro cualquiera. Ya vimos que no piensa en la jubilación, sino en seguir navegando mientras pueda en el Santa Eulàlia, recibiendo a bordo y haciendo disfrutar de la navegación en un velero histórico a los turistas y escolares que visitan diariamente lo que en el Museo Marítimo de Barcelona denominan su "Extensión en el agua".

Los casos de Ramon y Josep no pueden extrapolarse ni mucho menos al tercio de empleados del sector pesquero que perdieron sus puestos de trabajo en los últimos años. Pero sí nos resultan útiles para pensar sobre lo que está sucediendo y preguntarnos hacia dónde puede evolucionar la situación actual. En primer lugar podemos preguntarnos quiénes son los pescadores de ese tercio, capaces de reconvertir su actividad hacia el patrimonio marítimo. Sin duda la respuesta es aquellos que dispongan del necesario capital social y capital cultural para dar el salto. En segundo lugar podemos preguntarnos cuáles serán las consecuencias de la patrimonialización del ámbito marítimo-pesquero sobre la pesca a mediano plazo. Aquí sí que la pregunta, más que respuestas claras, ofrece nuevos interrogantes. De entrada se nos plantea una disyuntiva. Puede que las acciones de patrimonialización como las que realizan Ramon y Josep, así como la gran cantidad de actividades que se organizan las poblaciones de la costa en torno al patrimonio marítimo-pesquero por parte de asociaciones, ayuntamientos y museos, consigan sensibilizar a la población y a los propios pescadores sobre las problemáticas ambientales que afectan gravemente a los ecosistemas marinos, y de la necesidad apremiante de actuar para revertir la degradación del medio natural. Puede incluso que estas acciones logren convencer a más personas, acrecentando a través de la sensibilización el porcentaje de consumidores éticos y de turistas responsables. Pero también puede producirse el efecto contrario, es decir, que la patrimonialización no sea más que una forma de reinventar el turismo para renovar el modelo turístico de las cinco eses: Sun, Sea, Sand, Sex \& Sangria (Jiménez y Prats, 2006, p. 157) que lleva años dando síntomas de agotamiento en Cataluña y así seguir con una dinámica de sostenido crecimiento turístico en la costa, lo que probablemente llevaría en la práctica a la extinción de la pesca. Pero si la hipótesis acertada fuera la primera, en ese caso podríamos responder que sí a la pregunta de Perejaume, que un pescador que ha sido convertido al turismo podrá volver a ser pescador. Contemplando el fenómeno desde una perspectiva histórica, parece que esto sería lo más coherente con las oscilaciones que han caracterizado el contingente de pescadores en Cataluña a lo largo de la historia, como mostró Alfons Garrido (2012) en su tesis doctoral. Pero la cuestión, de momento, está en el aire.

Será importante en los años venideros seguir atentos a las relaciones entre pesca y turismo, tema que de hecho fue ya una de las primeras preocupaciones de la antropología de la pesca en Cataluña de la mano de Oriol Pi i Sunyer (1977a). Y añadir, a este binomio, un fenómeno nuevo: la patrimonialización del ámbito marítimo y particularmente la emergencia del patrimonio marítimo intangible. El turismo es uno de los fenómenos más favorecidos por la globalización (Meetham, 2001; Wahab y Cooper, 2001). Noel Salazar $(2005,2012)$ describe el turismo como uno de los estandartes de la globalización, poniendo énfasis en el hecho de que, en relación al turismo, lo global y lo local se encuentran estrechamente entrelazados. Y aquí es donde entra en juego el patrimonio cultural como instrumento para la creación de localidad (Frigolé y Roigé, 2006; Prats, 2006). Todo ello está dando lugar a mutaciones en la pesca artesanal tal y como la hemos conocido en las últimas décadas. Los efectos de la globalización sobre la pesca artesanal serán sin duda profundos y duraderos. 


\section{Referencias citadas}

Alberich, N. (2001). La feina d'en terra de la gent de mar: les remendadores de Cambrils. Quaderns de l'Institut Català d'Antropologia, 15-16, 89-102.

Alegret, J. L. (1986). Els armelladers de Palamós. Girona: Diputació de Girona.

Alegret, J. L. (1998). Property Rights, Regulatory Measures and Strategic Responses among the Fishermen of Catalonia. En Crean, K. y Symes, D. (Eds.). Fisheries Management in Crisis (pp. 175-187). Oxford: Fishing New Books - Blackwell.

Alegret, J. L. (1999a). Gestión comunitaria, cogestión y mercado. La evolución histórica de la gestión de la pesca en el Mediterráneo español. En VIII Congreso de Antropología (pp. 109-124). Santiago de Compostela: Asociación Galega de Antropoloxia.

Alegret, J. L. (1999b). Space, Resources and History: The Social Dimension of Fisheries in the Southwest Mediterranean. En Europe's Southern Waters: Management Issues and Practice. Oxford (GBR): Fishing New Books - Blackwell.

Alegret, J. L. (2002). L'activitat pesquera a Catalunya. La proa Diari del Baix Empordà, 35-39.

Alegret, J. L. (2016a). Un proyecto de revalorización del patrimonio cultural del pescado: El Espai del Peix de Palamós. Quederns Blaus, 31. Palamós: Càtedra d'Estudis Marítims.

Alegret, J. L. (2016b). Les cofradies de pescadors a Espanya. En Sánchez, J. L. (Ed.). La pesca a la Mediterrània. Alacant: Publicacions de la Universitat d'Alacant.

Alegret, J. L. y Garrido, A. (2008). Historia del puerto pesquero de Palamós: migraciones, asociacionismo y desarrollo. AREAS. Revista internacional de Ciencias Sociales, $27,27-40$.

Alegret, J. L. y Martí, M. (2003). La pesca en blanc i negre. En VV.AA. La pesca Catalunya (pp. 11-65). Barcelona: Angle Editorial.

Alegret, J. L. y Nadal, B. (1987). Les confraries de pescadors. La dimensió social de la pesca a Catalunya. Direcció Ge- neral de Pesca Marítima. Departament d'agricultura, ramaderia i pesca. Generalitat de Catalunya.

Amades, J. [1936] (2000). Mitologia de la mar. Tarragona: El Mèdol.

Andreu, A. (1981). La pesca en las albuferas del delta del Ebro. En Actas del II Congreso de antropología (pp. 303314). Madrid: Centro Nacional de Información Artística, Arqueológica y Etnológica.

Andreu, A. (1985). El proceso de apropiación de un espacio lacustre. Actas del III Congreso de Antropología. Donosti: Universidad del País Vasco.

Barbaza, Y. (1988). El paisatge humà de la Costa Brava. Barcelona: Edicions 62.

Bas, C. y Camprubí, R. (1980). La pesca a Catalunya. Barcelona: Edicions Destino.

Beorlegui, D. y Sotes, J. (2009). La crisis ecológica: el caso de la pesca en Gandía. En Ibarra, A. (Coord.). No es país para jóvenes. Encuentro de Jóvenes Investigadores en Historia Contemporánea. Granada: Instituto Valentín Foronda.

Böcker, R. (2013). Desarrollo económico y actividad pesquera en el sur de Cataluña. Revista Internacional de Organizaciones, 6, 45-63.

Boissevain, J. (1992). Revitalizing European Rituals. London: Routledge.

Braudel, F. (1949). La Méditerranée et le monde méditerranéen a l'époque de Philippe II. París: Armand Colin.

Breton, F. (1987). Características de la flota a Catalunya: Crítica de las categorías de flota industrial y flota artesanal a la luz de un ejemplo catalán. Jornadas sobre Economía y Sociología de las Comunidades Pesqueras. 20, 21 y 22 de mayo de 1987. Universidad de Santiago de Compostela. Madrid: Ministerio de Agricultura, Pesca y Alimentación.

Breton, F., Lladó, F. y Badia, A. (1992). Femmes pêcheurs et femmes de pêcheurs à la Barceloneta (Catalogne). Anthropologie Maritime, 4, 41-62.

Carbonell, E. (2013). El patrimonio inmaterial de las comunidades de Pescadores: Reflexiones desde Catalunya. Argos: Revista do Museu Maritimo de Ílhavo, 1, 29-38. 
Carbonell, E. (2014). Opportunities and Contradictions in Maritime Heritage and Small-scale Fishing. A Case Study of Catalonia. Collegium Antropologicum, 38(1), 289-296.

Carbonell, E. (2015). What is a Traditional Boat? The Continuity of Catalan Traditional Boats. En Lira, S., Amoeda, R. y Pinheiro, C. (Eds.). Shanring Cultures 2015. Proceedings of the $4^{\text {th }}$ International Conference on Intangible Heritage (pp. 339-348). Barcelos, Portugal: Green Lines Institute for Sustainable Development.

Carbonell, E. y Domènech, G. (2017). Reconversiones patrimoniales en el litoral noreste catalán: el paisaje cultural del pasado marítimo-pesquero. En Nadal, J. (Ed.). La ciudad y el mar. La patrimonialización de las ciudades portuarias (pp. 241-258). Girona: ICRPC.

Carbonell, E., Doñate, M. y Romero, P. (2011). Ser o no ser de mar. Arenys: Història, Patrimoni i Antropologia Maritima. Girona: Institut Català de Recerca en Patrimoni Cultural.

Crean, K. y Symes, D. (Eds.) (1996). Fisheries Management in Crisis. Oxford: Blackwell Science - Fishing New Books.

De la Cruz, R. (2012). Turismo, pesca y gestión de recursos. Aportaciones desde la Restringa y L'Estartit. Madrid: Ministerio de Educación, Cultura y Deporte.

Delgado, M. (2005). Elogi del vianant: del "model Barcelona" a la Barcelona real. Barcelona: Edicions de 1984.

Florido Del Corral, D. (2004). La pesca en Andalucía. Factores globales y locales de un proceso de crisis. Sevilla: Fundación José Manuel Lara.

Freire, J. y García-Allut, A. (2000). Socioeconomic and biological causes of management failures in European artisanal fisheries: the case of Galicia (NW Spain). $M a-$ rine Policy, 24(5), 375-384. DOI: 10.1016/S0308597X(00)00013-0.

Frigolé, J. y Roigé, X. (Coords.) (2006). Globalización y localidad: Perspectiva etnográfica. Barcelona: Publicacions de la UB.

Garay, L. A. y Cànoves, G. (2010). Un anàlisis del desarrollo turístico en Cataluña a través del ciclo de evolución del destino turístico. Boletín de la Asociación de Geógrafos Españoles, 52, 43-58.
Garrido, A. (2012). La pesca al Cap de Creus a l'època moderna (segles XVI-XVIII): organització, gestió $i$ conflictes per l'accés als recursos pesquers (Tesis doctoral). Universitat de Girona, Girona.

Gascón, J. y Cañada, E. (2005). Viajar a todo tren: Turismo, desarrollo y sostenibilidad. Barcelona: Icaria.

Generalitat de Catalunya (2013). Catalunya turistica en xifres 2013. Barcelona: Direcció General de Turisme, Departament d'Empresa i Ocupació.

Gracia, P. (2016). El turisme pesquer. En Sánchez, J. L. (Ed.). La pesca a la Mediterrània (pp.123-126). Alacant: Publicacions de la Universitat d'Alacant.

Greenpeace (2013). Empleo a bordo. Análisis del empleo en el sector pesquero español y su impacto socioeconómico. Madrid: Greenpeace España.

Jiménez, S. y Prats, L. (2006). El turismo en Cataluña: evolución histórica y retos de futuro. Pasos. Revista de turismo y patrimonio cultural, 4(2), 153-174. DOI: 10.25145

Kammerer, N. (2014). Catalan Festival Culture, Identities, and Independentism. Quaderns de l'Institut Català d'Antropologia, 19(2), 58-78.

Lleonart, J. (1996). La pesca a Catalunya i la seva gestió. Butll. Inst. Cat. Hist. Nat., 64, 135-158.

Llovet, L. (1984). La Catalunya marítima a la segonameitat del segle XVIII. La revista de matrícules Luís Muñóz de Guzmán (1786). Actes del Primer Congrés d'Història Moderna de Catalunya (pp. 711-720). Barcelona: Edicions de la Universitat de Barcelona.

MacCannell, D. (1976). The Tourist: A New Theory of the Leisure Class. New York: Schocken Books.

Mas, X. (1994). Memorial dels pescadors i els peixos. Mataró: Caixa d'Estalvis Laietana.

McGoodwin, J. R. (1990). Crisis in the world's fisheries people, problems and policies. Stanford: Stanford University Press.

Meetham, K. (2001). Tourims in Global Society: Place, Culture, Consumption. New York: Palgrave.

Nadel-Klein, J. (2003). Fishing for Heritage. Modernity and Loss Along the Scottish Coast. Oxford: Berg. 
Newholm, T. y Shaw, D. (2007). Studying the ethical consumer: A review of research, Journal of Consumer Behaviour, 6(5), 253-270. DOI: 10.1002/cb.225

Palou, S. (2012). Barcelona, destinació turistica. Un segle d’imatges i promoció pública. Barcelona: Edicions Vitel.la.

Palou, S. (2017). 'Maritimidad reinventada'. Patrimonio y turismo en los puertos de Roses y Palamós. En Nadal, J. (Ed.). La ciudad y el mar. La patrimonialización de las ciudades portuarias (pp. 259-280). Girona: ICRPC.

Pascual, J. (2004). Las investigaciones sobre la pesca en Canarias: entre las reservas marinas y las nuevas formas de pescaturismo. Pasos. Revista de Turismo y Patrimonio Cultural, 2(2), 295-306. DOI: 10.25145

Pascual, J. y Alegret, J. L. (2004). Estado actual de la antropología de la pesca en España. Quaderns Blaus, QB010. Palamós: Cátedra d'Estudis Marítims de la Universitat de Girona i Museu de la Pesca.

Pi i Sunyer, O. (1977a). Through Native Eyes: Tourists and Tourism in a Catalan Maritime Community. En Smith, V. L. (Ed.). Hosts and Guests. The Anthropology of Tourism (pp. 149-155). Philadelphia, PA: University of Pennsylvania Press.

Pi i Sunyer, O. (1977b). Two Stages of Technological Change in a Catalan Fishing Community. En Smith, E. M. (Ed.). Those who live from the sea: A Study in Maritime Anthropology. St. Paul: West.

Prat, J., Martínez, U. y Contreras, J. (1991). Antropología de los pueblos de España. Madrid: Taurus.

Prats, L. (1997). Antropología y patrimonio. Barcelona: Ariel.

Prats, L. (2006). La mercantilización del patrimonio: entre la economía turística y las representaciones identitarias. PH Boletín del Instituto Andaluz de Patrimonio Histórico, 58, 72-80.
Pujol, E. y Carbonell, E. (2009). Records d'una dona de mar. Palamós: Fundació Pro-Mediterrània.

Roig, E. (1927). La pesca a Catalunya. Barcelona: Barcino.

Ros, J. (2016). La producción primària, la base de tot. En Sánchez, J. L. (Ed.). La pesca a la Mediterrània (pp. 1118). Alacant: Publicacions de la Universitat d'Alacant.

Salazar, N. (2005). Más allá de la Globalización. La "Glocalización” del Turismo. Política y Sociedad, 42(1), 135-149.

Salazar, N. (2012). Envisioning Eden. Mobilizing Imaginaries in Tourism and Beyond. Oxford: Berghahn Books.

Saló, A. (2006) Estudi sobre l'habitatge a la demarcació de Girona (Tesis doctoral). Universitat de Girona, Girona.

Santana, A. y Pascual, J. (2003). Pesca y turismo: conflictos, sinergias y usos múltiples en Canarias. Boletín del Instituto Andaluz de Patrimonio Histórico, PH44, 86-97.

Torra, E. y Carbonell, E. (2015). Les dones i el mar. Barcelona: Museu Marítim de Barcelona.

Tudela, S. (2002). Evaluación de la huella ecológica en la costa central catalana. En Lleonart, J. y Sánchez, J. L. (Coords.). Séptima reunión del Foro Científico sobre la pesca española en el Mediterráneo [actas] (pp. 109-110). Alacant: Publicacions de la Universitat d'Alacant.

Vaczi, M. (2016). Catalonia's Human Towers: Nationalism, Associational Culture, and the Politics of Performance. American Ethnologist, 43(2), 353-368. DOI: 10.1111/ amet. 12310

Varela, M. y Ojeda, C. (2010). La pesca en España: Una lección no aprendida. Estudio de las pesquerias y recomendaciones ante la reforma de la Politica Pesquera Común. Madrid: Greenpeace.

Wahab, S. y Cooper, C. (2001). Tourism in the Age of Globalisation. London: Routledge. 